\title{
SÍNTESIS Y CARACTERIZACIÓN RMN 1D Y 2D DE DERIVADOS SULFURADOS DE LA 1- FENIL-3- METIL-2-PIRAZOLÍN-5-ONA Y SU POTENCIAL CAPACIDAD LIGANTE
}

\author{
Sergio Zamorano ${ }^{1 *}$, Juan Camus ${ }^{1}$
}

\begin{abstract}
RESUMEN
En este trabajo se presenta el resultado de la síntesis del 4-ditiocarboxilato de hexil-1-fenil3-metil-2-pirazolin-5-ona $\left(\mathrm{PirC}_{6}\right)$ y del 4-ditiocarboxilato de dodecil-1-fenil-3-metil-2pirazolin-5-ona $\left(\mathrm{PirC}_{12}\right)$. Se caracterizan los productos obtenidos usando el microanálisis, la espectroscopía infrarroja, la espectroscopía $\mathrm{RMN}$ de $\mathrm{H}^{1}$ y $\mathrm{C}^{13}$ y la $\mathrm{RMN}$ en dos dimensiones (HSQC y HMBC) para determinar sus estructuras. Se estudia su potencial capacidad ligante en la formación de compuestos de coordinación con el $\mathrm{UO}_{2}{ }^{2+}$.

Palabras clave: Derivados de la pirazolona, espectroscopía RMN 1D y 2D

\section{SYNTHESIS AND CHARACTERIZATION NMR 1D AND 2D OF THE SULPHURATED DERIVATIVES OF THE 1-PHENYL- 3-METHYL-2-PIRAZOLINE-5-ONA AND THEIR LIGAND POTENCIAL CAPACITY}

\begin{abstract}
In this work the result of the synthesis of the 4- hexil-dithiocarboxilate-1-phenil-3-methyl2-pirazolin-5-ona( $\left.\mathrm{PirC}_{6}\right)$, and 4-dithiocarboxilate of dodecil-1-phenil-3-methyl-2-pirazolin5 -ona $\left(\mathrm{PirC}_{12}\right)$, is presented and the products are characterized, using the microanalysis , the infrared spectroscopy, the spectroscopy of NMR of $\mathrm{H}^{1}$ and $\mathrm{C}^{13}$ and the NMR in two dimensions (HSQC and HMBC), in order to determine their structures. In addition, the ligand potential capacity of these products are studied in order to produce coordination compounds with the uranil $\left(\mathrm{UO}_{2}^{2+}\right)$.
\end{abstract}

Key words: Pyrazoline derivatives, 1D and 2D NMR spectroscopy

\section{INTRODUCCIÓN}

En publicaciones anteriores expusimos la síntesis y caracterización de moléculas orgánicas y bases de Schiff; aplicamos una variedad de experimentos de la RMN 1D y 2D, para determinar la estructura de las moléculas orgánicas sintetizadas ${ }^{1-2}$. Diversos investigadores han extendido el empleo de estas técnicas a la investigación estructural de las moléculas organometálicas ${ }^{3}$. Específicamente, existe una importante contribución en el campo de la química de los compuestos de coordinación de derivados de la pirazolona que son bases de Schiff, y actúan como agentes secuestrantes del uranilo, en la formación de complejos dioxouranio (VI), octacoordinados mono- y binucleares ${ }^{4}$. El uranio, en su estado de oxidación +6 , presente en el ion uranilo $\left(\mathrm{UO}_{2}^{2+}\right)$, tiene una gran afinidad para formar complejos estables con una variedad de ligandos que contienen átomos donores de oxígeno, nitrógeno y azufre 5 .

${ }^{1}$ Facultad de Ciencias Naturales y Exactas. Universidad de Playa Ancha, Carvallo No270, Playa Ancha, Valparaíso, Chile 
La RMN 1D y 2D ha resultado ser una herramienta muy útil en el estudio de las pirazolonas, permitiendo determinar la estructura de diversos derivados de este compuesto ${ }^{6-10}$.

Desde el punto de vista de la síntesis para obtener los derivados sulfurados de la pirazolona, esta se hace reaccionar con $\mathrm{CS}_{2}$ y luego con un halogenuro de alquilo, en medio alcalinoalcohólico ${ }^{6}$ para reemplazar un hidrógeno del carbono 4 , por una cadena carbonatada alifática, de acuerdo con la reacción presentada en la figura 1.

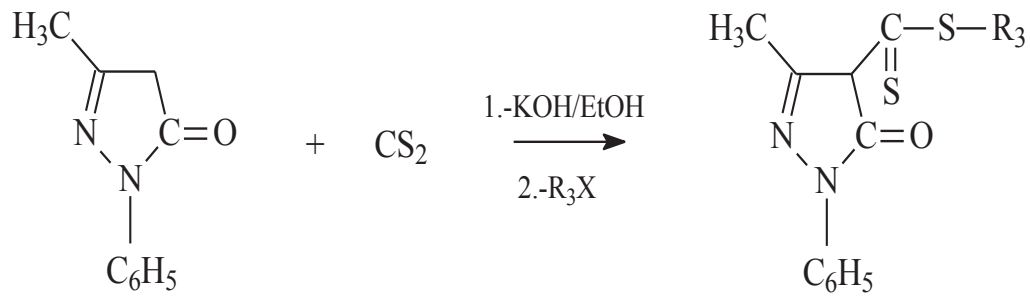

Figura 1. Síntesis de derivados sulfurados de la pirazolona

Al sustituir $\mathrm{R}$ mediante la adición del 1-bromododecano se obtiene el $\mathrm{PirC}_{12}$, con el 1-bromohexano el $\mathrm{PirC}_{6}$, con el cloroformiato de butilo el $\mathrm{PirC}_{4}$ y con el cloroformiato de fenilo7 el Pir-fenil. La RMN 1D y 2D nos permitió elucidar las estructuras ${ }^{8,9}$ de cada uno de los productos sintetizados, siguiendo las pautas de la bibliografía especializada ${ }^{10}$ :

- Estudio de espectro previo de $1 \mathrm{H}$ que permite descartar la existencia de impurezas que provocan superposiciones en el espectro.

- Determinación del solvente más adecuado, para seleccionar la mejor solubilidad posible y evitar interacciones o interferencias con el solvente.

- Determinación de los desplazamientos químicos de 13C.

- Clasificación de las zonas espectrales ( aromáticas, dobles enlaces, alifáticas, etc.).

- Determinación previa de carbonos cuaternarios ( con experimentos DEPT y/o con HSQC).

\section{Materiales y métodos}

\section{PARTE EXPERIMENTAL}

\section{Síntesis del PirC}

En $200 \mathrm{ml}$ de etanol se disuelve $20 \mathrm{mmol}$ (3,48 g) de 1-fenil-3-metil-2-pirazolín-5-ona. A la solución resultante se agrega una solución de $11 \mathrm{mmol}(0,62 \mathrm{~g})$ de $\mathrm{KOH}$ en $50 \mathrm{ml}$ de etanol. Se deja a reflujo durante 1 hora. La solución resultante es de color amarilla; se deja enfriar y se agrega $4 \mathrm{ml} \mathrm{de} \mathrm{CS}_{2}$. Posteriormente se somete a reflujo durante 8 horas. Se agregan 22 mmol (3,62 g) de 1-bromohexano y se somete a reflujo otras 8 horas. La solución resultante tiene un color amarillo-anaranjado. Se enfría la solución resultante en refrigerador a $5^{\circ} \mathrm{C}$ durante una semana. Se obtiene cristales de color amarillo.

\section{Síntesis del PirC ${ }_{12}$}

En $200 \mathrm{ml}$ de etanol se disuelve $20 \mathrm{mmol}$ (3,48 g) 1-fenil-3-metil-2-pirazolín-5-ona, luego se agrega una solución de $11 \mathrm{mmol}(0,62)$ de $\mathrm{KOH}$ en $50 \mathrm{ml}$ de etanol. Se somete a reflujo 
durante 1 hora. Se enfría la solución resultante y se agrega $1 \mathrm{ml}$ de $\mathrm{CS}_{2}$. La solución obtenida es de color amarillo pálido la que se somete a reflujo durante 1 hora. Posteriormente, se agregan $22 \mathrm{mmol}(5,46 \mathrm{~g})$ de 1-bromododecano y se deja a reflujo otras 8 horas. Se obtienen cristales de color amarillo-naranja

\section{Síntesis del complejo [ $\left.\mathrm{UO}_{2}\left(\mathrm{PirC}_{6}\right)\right]$}

Se disuelven $100 \mathrm{mg}$ de PirC6 en $50 \mathrm{ml}$ de alcohol metílico absoluto. A la solución resultante se agrega $63,5 \mathrm{mg}$ de acetato de uranilo, disueltos en $50 \mathrm{ml}$ de metanol. La solución resultante se deja a reflujo durante 2 horas. Se obtiene una solución anaranjada, que se deja reposar en el refrigerador a $5^{\circ} \mathrm{C}$ durante una semana. Se obtienen cristales de color naranja oscuro.

\section{RESULTADOS Y DISCUSIÓN}

El resultado del análisis químico elemental se obtuvo usando un equipo Fison EA-1108 (CHNS-O).

En el caso del PirC $\mathbf{~}_{6}$, se tiene que los porcentajes de carbono, hidrógeno, nitrógeno y azufre, teórico/experimental son: $61,12 / 60,42 ; 6,59 / 6,66 ; 8,45 / 8,36 ; 19,20 / 19,84$, respectivamente. En el caso del complejo $\mathrm{UO}_{2}^{++}$con PirC $_{6}$, se tiene que los porcentajes de carbono, hidrógeno, nitrógeno y azufre, teórico/experimental son: 43,60/42,24; 4,50/4,70; 6,08/5,88 y $13,70 / 13,96$, respectivamente.

En el caso del PirC $\mathrm{C}_{12}$, se tiene que los porcentajes de carbono, hidrógeno, nitrógeno y azufre, teórico/experimental son: $65,84 / 64,12 ; 8,13 / 8,07 ; 6,69 / 6,84$ y 15,54/15,90, respectivamente. Los resultados de la espectroscopía IR, registrados en un espectrofotómetro IR-Brucker FTIR con ventanas de $\mathrm{KBr}$ en rango $4000-200 \mathrm{~cm}^{-1}$, son los siguientes:

En el caso del PirC $_{12}$ : Las bandas más importantes se encuentran agrupadas en el anillo del pirazol que aparecen a los $1551,4 \mathrm{~cm}^{-1}$, la parte aromática aparece a los 3061,8 y 753,2 $\mathrm{cm}^{-1}$. La parte alifática aparece a los 2922 y $2852 \mathrm{~cm}^{-1}$. La parte sulfurada aparece en dos bandas a los 1599,7 y $1064,7 \mathrm{~cm}^{-1}$. La banda $\mathrm{OH}-$ se encuentra a $3448,2 \mathrm{~cm}^{-1}$.

En el caso del PirC $_{6}$ : Las bandas más importantes se encuentran agrupadas en el anillo del pirazol que aparecen a $\operatorname{los} 1556,1 \mathrm{~cm}^{-1}$, la parte aromática aparece a los $3064,1 \mathrm{y} 755,5 \mathrm{~cm}^{-1}$. La parte alifática aparece a los 2954,2;2927,9 $\mathrm{cm}^{-1}$ y $2853,7 \mathrm{~cm}^{-1}$. La parte sulfurada aparece a los $1057,8 \mathrm{~cm}^{-1}$ La banda del $\mathrm{OH}-$ aparece a los $3458,8 \mathrm{~cm}^{-1}$.

Los espectros en una dimensión de $\mathrm{RMN}^{1} \mathrm{H}$ y $\mathrm{RMN}{ }^{13} \mathrm{C}$ del $\mathrm{PirC}_{6}$ y del $\mathrm{PirC}_{12}$ muestran las siguientes señales:

PirC : $_{6} \mathrm{RMN}{ }^{1} \mathrm{H}\left(\mathrm{ppm}, 400 \mathrm{Mhz}, \mathrm{CDCl}_{3}\right): \delta$ 0,88 (3H,m); 1,27 (4H,m); 1,48 (2H,m); 1,80 $(2 \mathrm{H}, \mathrm{m}) ; 2,67(3 \mathrm{H}, \mathrm{s}) ; 3,39(2 \mathrm{H}, \mathrm{m}) ; 7,33(1 \mathrm{H}, \mathrm{m}) ; 7,47(2 \mathrm{H}, \mathrm{m}) ; 7,82(2 \mathrm{H}, \mathrm{m}) ; 12,56(1 \mathrm{H}, \mathrm{s})$

PirC $_{12}: \mathrm{RMN}^{1} \mathrm{H}\left(\mathrm{ppm}, 400 \mathrm{Mhz}, \mathrm{CDCl}_{3}\right): \delta$ 0,88 (3H,m); 1,27 (24H,m); 1,48 (2H,m); 1,80 $(2 \mathrm{H}, \mathrm{m}) ; 2,67(3 \mathrm{H}, \mathrm{s}) ; 3,39(2 \mathrm{H}, \mathrm{m}) ; 7,31(1 \mathrm{H}, \mathrm{m}) ; 7,47(2 \mathrm{H}, \mathrm{m}) ; 7,80(2 \mathrm{H}, \mathrm{m}) ; 13,72(1 \mathrm{H}, \mathrm{s})$

PirC $_{6}: \mathrm{RMN}^{13} \mathrm{C}\left(\mathrm{ppm}, 400 \mathrm{Mhz}, \mathrm{CDCl}_{3}\right):$ 14,31(1C,s); 18,26 (1C,s); 22,66(1C,s); 27,98(1C,s); $29,03(1 \mathrm{C}, \mathrm{s}) ; \quad 31,66(1 \mathrm{C}, \mathrm{s}) ; \quad 33,77(1 \mathrm{C}, \mathrm{s}) ; \quad 111,40(1 \mathrm{C}, \mathrm{s}, \mathrm{q}) ; \quad 121,68(2 \mathrm{C}, \mathrm{s}) ; \quad 126,91(1 \mathrm{C}, \mathrm{s})$; $129,32(2 \mathrm{C}, \mathrm{s}) ; 137,06(1 \mathrm{C}, \mathrm{s}, \mathrm{q}) ; 146,89(1 \mathrm{C}, \mathrm{s}, \mathrm{q}) ; 157,75(1 \mathrm{C}, \mathrm{s}, \mathrm{q}) ; 213,80(1 \mathrm{C}, \mathrm{s}, \mathrm{q})$

$\operatorname{PirC}_{12}: \mathrm{RMN}^{13} \mathrm{C}\left(\mathrm{ppm}, 400 \mathrm{Mhz}, \mathrm{CDCl}_{3}\right): 14,14(1 \mathrm{C}, \mathrm{s}) ; 18,31(1 \mathrm{C}, \mathrm{s}) ; 28,10(1 \mathrm{C}, \mathrm{s}) ; 29,15(1 \mathrm{C}, \mathrm{s})$; 29,31-29,85(6C,s); 32,29(1C,s); 34,10(1C,s); 111,48(1C,s,q); 122,00(2C,s); 127,04(1C,s); $129,13(2 \mathrm{C}, \mathrm{s}) ; 137,32(1 \mathrm{C}, \mathrm{s}, \mathrm{q}) ; 147,02(1 \mathrm{C}, \mathrm{s}, \mathrm{q}) ; 157,83(1 \mathrm{C}, \mathrm{s}, \mathrm{q}) ; 214,10(1 \mathrm{C}, \mathrm{s}, \mathrm{q})$ 
De los espectros RMN C ${ }^{13}$ y DEPT 135 se deduce cuál carbono de las moléculas $\operatorname{PirC}_{6} \mathrm{y}$ $\mathrm{PirC}_{12} \mathrm{~s}$ cuaternario, ya que las diferencias entre las señales producidas en los espectros DEPT 135 y RMN C ${ }^{13}$ son las señales de los carbonos cuaternarios, las cuales no se presenta en el espectro DEPT 135; de aquí determinamos la presencia de cinco señales correspondientes a carbonos de este tipo que no se enlazan directamente al hidrógeno; tienen los siguientes desplazamientos: 157,75; 146,89; 137,21; 213,75; 111,67 ppm en PirC6. En el caso del PirC ${ }_{12}$ se presenta las señales 214,$03 ; 157,84 ; 146,95 ; 137,41 ; 111,49$ ppm.

De acuerdo a los espectros $\mathrm{RMN} \mathrm{H} \mathrm{H}^{1}$, RMN $\mathrm{C}^{13}$, experimento DEPT 135 y espectros bidimensionales se realizó la asignación para cada uno de los núcleos de carbono e hidrógeno de la estructura.

Para el $\mathrm{PirC}_{6}$ los carbonos se marcan de manera correlativa con números del 1 al 17 y los hidrógenos se designan con letras minúsculas de "a" a la "l"; en cambio, para el PirC $_{12}$ los carbonos se rotulan del 1 al 23 y los hidrógenos de "a" a " $r$ ", como se muestra en la figura 2. El espectro bidimensional HSQC que correlaciona hidrógenos con carbono que se encuentran enlazados directamente, en el caso del PirC6, se presenta en la figura 3. El espectro HMBC muestra correlaciones entre hidrógenos y carbono situados a una distancia de 2 o más enlaces.<smiles>CCCCCCSC(=S)c1c(C)nn(-c2ccccc2)c1O</smiles><smiles>[R][SH]Cc1c(C)nn(-c2ccccc2[2H])c1O</smiles>

Figura 2. Estructura y numeración de los derivados sulfurados de la pirazolona $\operatorname{PirC}_{6}$ y $\operatorname{PirC}_{12}$ 


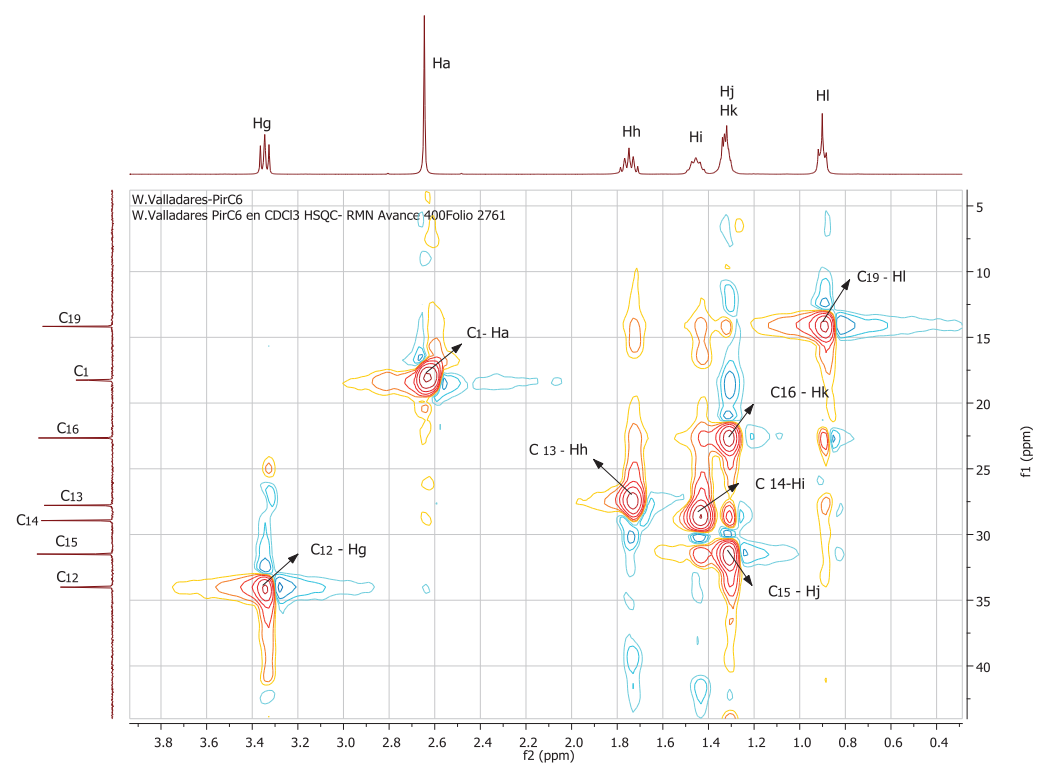

Figura 3. Diagrama de contorno $\mathrm{HSQC}$ del $\mathrm{PirC}_{6}$

El espectro $\mathrm{HMBC}$, que se muestra en las figuras 4 y 5, pone en evidencia la relación entre carbonos y protones que se encuentren a 2 ó 3 enlaces de distancia.

La figura 4 muestra aquellas señales que corresponden a las interacciones entre los carbonos $1,12,13,14,15$ y 16 con los protones $\mathbf{a}, \mathbf{h}, \mathbf{g}, \mathbf{j} \mathbf{y} \mathbf{l}$.

La señal para $\mathrm{C}_{1}$ y $\mathrm{H}_{\mathrm{a}}$ indica acoplamiento de estos, así también, la señal para $\mathrm{C}_{12}$ y $\mathrm{H}_{\mathrm{g}}$ indica acoplamiento entre ellos.

Para el $\mathrm{C}^{13}$ y el Hg la señal también indica acoplamiento, y además, se observa que el mismo protón posee una interacción con el carbono 12 .

La figura 5 muestra la ampliación del espectro $\mathrm{HMBC}$ de la zona aromática. Las señales correspondientes para el $\mathrm{C}_{4}, \mathrm{C}_{8} \mathrm{y} \mathrm{H}_{\mathrm{b}}, \mathrm{H}_{\mathrm{f}}$, indican el acoplamiento entre los carbonos y los protones; además de una interacción entre los protones y los carbonos opuestos; es decir, que el protón $\mathrm{b}$ interacciona con el carbono 8 y el protón $\mathrm{f}$ con el carbono 4 . El C3 también interacciona con este mismo par de protones.

Para los protones $\mathrm{Hc}$ y He existe una señal de acoplamiento con los carbonos $\mathrm{C}_{5}$ y $\mathrm{C}_{7}$, y otra que muestra la interacción de los protones con los carbonos alternos. Además, se encuentran las señales de interacción entre los protones antes nombrados con los carbonos $\mathrm{C}_{4}, \mathrm{C}_{8} \mathrm{y} \mathrm{C}_{3}$, los que se encuentran a 2 y 3 enlaces de distancia, respectivamente.

Por último, la figura 5 muestra el acoplamiento del $\mathrm{C}_{6}$ con el $\mathrm{H}_{\mathrm{b}} \mathrm{y} \mathrm{H}_{\mathrm{f}}$, que se encuentra a 3 enlaces de distancia y la interacción del protón $\mathrm{H}_{\mathrm{d}}$ con $\mathrm{C}_{4} \mathrm{y}_{8}$. Además, muestra las interacciones del carbono $\mathrm{C}_{3}$ con los protones $\mathrm{Hc}$ y He, que se encuentran a 3 enlaces de distancia y con los protones vecinos $\mathrm{H}_{\mathrm{b}} \mathrm{y} \mathrm{H}_{\mathrm{f}}$. 


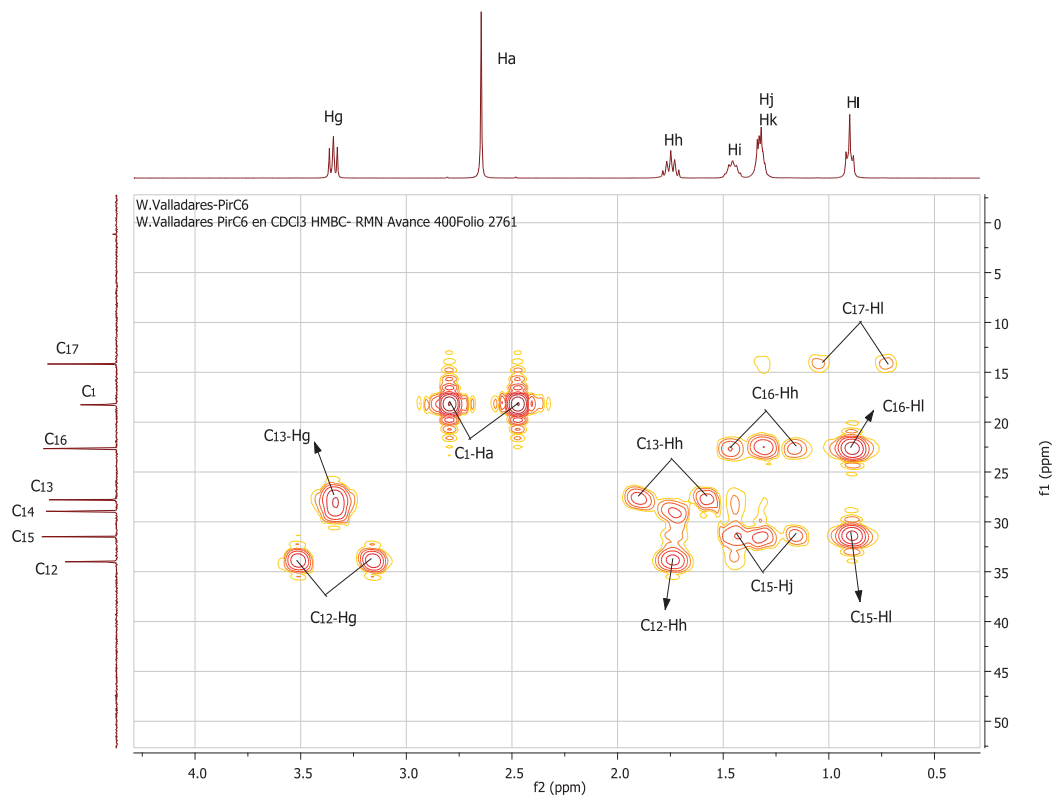

Figura 4. Diagrama de contorno $\mathrm{HMBC}$ del $\mathrm{PirC}_{6}$

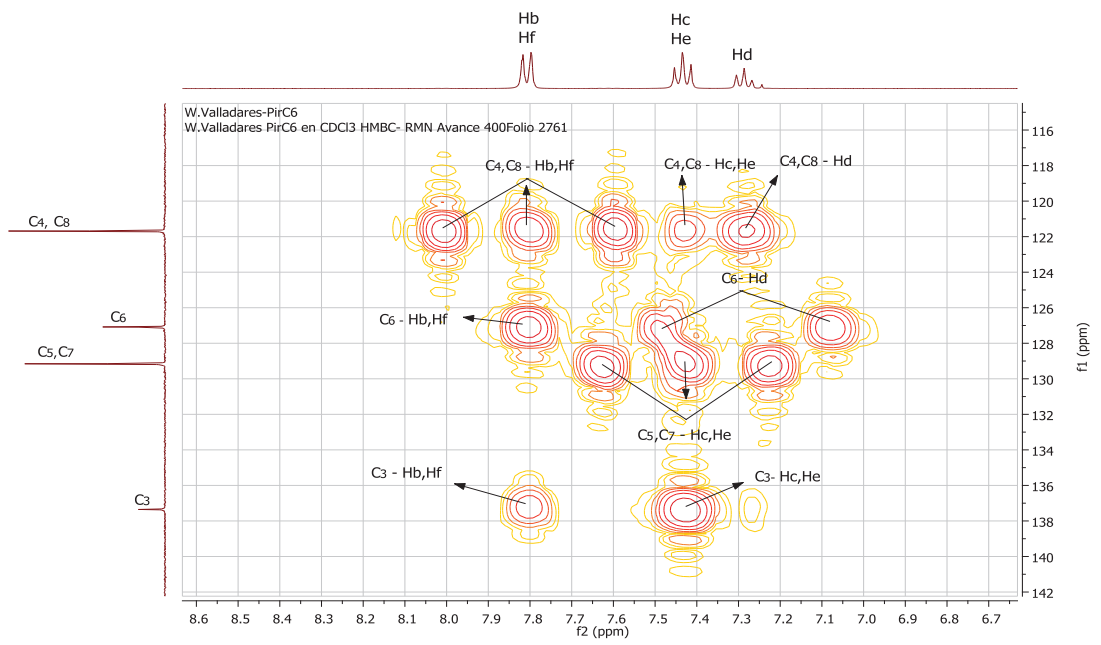

Figura 5. Diagrama de contorno $\mathrm{HMBC}$ del $\mathrm{PirC}_{6}$, zona aromática. 
La tabla 1 muestra aquellas señales que corresponden a las interacciones entre los carbonos 18 al 23 de la cadena alifática en $\operatorname{PirC}_{12}$, con los protones $\mathbf{k}, \mathbf{l}, \mathbf{m}, \mathbf{n}, \mathbf{o}, \mathbf{p}$ y q. Se observa que la interacción a dos o tres enlaces en todos los casos se produce a 1,270 ppm. Así, por ejemplo, la señal para $\mathrm{C}_{18}$ y los protones $\mathrm{H}_{\mathrm{k}}, \mathrm{H}_{\mathrm{l}}, \mathrm{H}_{\mathrm{n}} \mathrm{y} \mathrm{H}_{\mathrm{o}}$ indica acoplamiento de estos a 1,270 ppm.

Tabla1. Señales de RMN bidimencional H-C, directo (Hd) y HMBC (interacción a dos y tres enlaces, $\mathrm{Hi}$ ) para Pir $\mathrm{C}_{12}$

\begin{tabular}{|c|c|c|c|}
\hline $\mathrm{C}$ & $\mathrm{ppm}$ & $\mathrm{H}_{\mathrm{d}}, \mathrm{ppm}$ & $\mathrm{H}_{\mathrm{i}}, \mathrm{ppm}$ \\
\hline 18 & 29,785 & (m) 1,270 & $\begin{array}{l}(\mathrm{k}, \mathrm{l}, \mathrm{n}, \mathrm{o}) \\
1,270\end{array}$ \\
\hline 19 & 29,555 & (n) 1,270 & $\begin{array}{l}(1, m, o, p) \\
1,270\end{array}$ \\
\hline 20 & 29,674 & (o) 1,270 & $\begin{array}{l}(m, n, p, q) \\
1,270\end{array}$ \\
\hline 21 & 32,135 & (p) 1,270 & $\begin{array}{l}(\mathrm{n}, \mathrm{o}, \mathrm{q}, \mathrm{r}) \\
1,270\end{array}$ \\
\hline 22 & 22,897 & (q) 1,270 & $\begin{array}{l}(\mathrm{o}, \mathrm{p}, \mathrm{r})) \\
1,270\end{array}$ \\
\hline 23 & 14,304 & (r) 0,882 & $\begin{array}{l}(\mathrm{q}, \mathrm{p}) \\
1,270\end{array}$ \\
\hline
\end{tabular}

En base a los resultados de la espectroscopía IR, donde se observa que en el espectro del $\mathrm{UO}_{2}$ (PirC-6) ${ }_{2}$ aparece un pico fuerte a los $926,6 \mathrm{~cm}^{-1}$ correspondiente al $\mathrm{U}=\mathrm{O}$ y el surgimiento de la banda pequeña a los $507,0 \mathrm{~cm}^{-1}$, atribuida al enlace entre el ligando y el uranilo U-O. De los resultados del análisis químico elemental, postulamos la formación de un compuesto de coordinación de tipo $\mathrm{UO}_{2}$ (PirC-6) cuya estructura podría esquematizarse de acuerdo a la figura 6 .

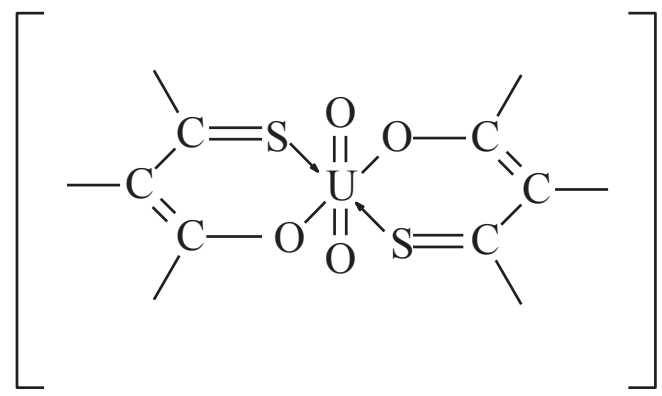

Figura 6. Coordinación del uranilo con ligandos oxígeno-sulfurados de la pirazolona

No se pudo realizar el espectro de $\mathrm{RMN}$ ni de $\mathrm{H}^{1}$ ni de $\mathrm{C}^{13}$ por la baja solubilidad del complejo en los solventes disponibles. 


\section{CONCLUSIONES}

Se sintetizó dos compuestos orgánicos derivados de la 1- fenil-3- metil-2-pirazolín--5-ona, los que fueron caracterizados mediante los espectros IR y de RMN mono y bidimensionales, además de la caracterización mediante el análisis químico elemental. Se sintetizó, además, un compuesto de coordinación al que se propuso una estructura tipo quelato, el que pudiera participar en reacciones con transferencia de electrones, por su especial configuración de tipo

$$
\left[\mathrm{UO}_{2}\left(\mathrm{O}_{2} \mathrm{~S}_{2}\right)\right]^{\mathrm{O}}
$$

\section{BIBLIOGRAFÍA}

1. S. Zamorano, J. Camus, A. Orellana, M.l Osorio. Caracterización de bases de Schiff derivadas de 2-aminofenol, usando RMN 1D y 2D. Rev. Soc. Quim. Perú.2010; 76 (2):187-193.

2. S. Zamorano, J. Camus. Determinación de la Estructura de Bases de Schiff derivadas 2-aminofenol nitro y fluor sustituidas, utilizando la RMN 1D y 2D. Rev. Soc. Quim. Perú. 2011; 77 (1):27-34 (2011).

3. E.Gaggelli, N. Gaggelli y G. Valensin. 2D NMR Methods for Structural Delineation of Copper(II) Complexes of Penicillin and Pilocarpine. Metal-Based Drugs. Volume 1 (1994), Issue 2-3, Pages 279-287 doi:10.1155/MBD.1994.279

4. R.C. Maurya et. al.Coordination chemistry of pyrazolone based Schiff bases relevant to uranyl sequestring agent: Synthesis, characterization and 3D molecular modeling of some octa-coordinate mono- and binuclear-dioxouranium(VI) complexes. Arabian Journal of Chemistry. Disponible online 5 February 2011. http://dx.doi.org/10.1016/j. bbr.2011.03.031

5. R.C. Maurya, et al. Synthesis and characterization of Mixed-Ligand Complexes of $\mathrm{Cu}$ (II), Ni (II), Co (II), Zn (II), Sm (III), and U (VI) O2, with a Schiff Base Derived from the Sulfa Drug Sulfamerazine and 2, 2'-Bipyridine. Synthesis and reactivity in inorganic and metal-organic chemistry, 2003; 33(5): 801-816.

6. V. Berrueta, J. Cáceres y L.Cerda. Seminario de Tesis para optar al título de Profesor de Química y al grado de Licenciado en Educación. Valparaíso, Chile. Universidad de Playa Ancha. 2009.

7. P. González. Seminario de Tesis para optar al título de Profesor de Química y al grado de Licenciado en Educación. Valparaíso, Chile. Universidad de Playa Ancha. 2009.

8. C. Soto. Seminario de Tesis para optar al título de Profesor de Química y al grado de Licenciado en Educación. Valparaíso, Chile. Universidad de Playa Ancha. 2010.

9. A. Osorio. Seminario de Tesis para optar al título de Profesor de Química y al grado de Licenciado en Educación. Valparaíso, Chile. Universidad de Playa Ancha. 2009.

10. A. Castillo, X. Muñoz. Seminario de Tesis para optar al título de Profesor de Química y al grado de Licenciado en Educación. Valparaíso, Chile Universidad de Playa Ancha. 1996.

11. H. Friebolin "Basic one-and two-dimensional NMR spectroscopy" Wiley-VCH, 1998. 
12. H. Dudderck, W. Dietrich y G. Toth" Elucidación Estructural por RMN" Ed. Springer. 3ra.ed. 2000.

13. M. Feliz y A. Molins. Determinación estructural. Utilización combinada de experimentos RMN. Universitat de Barcelona. Serveis Cientific Tecnics. Unitat de RMN.

http://www.rmn.ub.es/cat/cursos/material/docs/altres/dossier_estructral-Quinina.pdf http://ebookbrowsee.net/curso-rmn-pdf-d394184844. 